\title{
Rare microbes from diverse Earth biomes dominate community activity
}

\author{
Rohan Sachdeva*1,2, Barbara J. Campbell ${ }^{3}$, and John F. Heidelberg ${ }^{1,4,5}$
}

1. Department of Biological Sciences, University of Southern California, Los Angeles, CA 90089, USA

2. Innovative Genomics Institute, University of California, Berkeley, 94720, California, USA (current affiliation)

3. Department of Biological Sciences, Life Science Facility, Clemson University, Clemson, SC 29634, USA

4. Center for Dark Energy Biosphere Investigations, University of Southern California, Los Angeles, CA 90089, USA

5. Wrigley Institute for Environmental Studies, University of Southern California, Los Angeles, CA 90089, USA

*Corresponding author: Rohan Sachdeva (rohansach@berkeley.edu)

\begin{abstract}
Microbes are the Earth's most numerous organisms and are instrumental in driving major global biological and chemical processes. Microbial activity is a crucial component of all ecosystems, as microbes have the potential to control any major biochemical process. In recent years, considerable strides have been made in describing the community structure, i.e. diversity and abundance, of microbes from the Earth's major biomes. In virtually all environments studied, a
\end{abstract}


few highly abundant taxa dominate the structure of microbial communities. Still, microbial diversity is high and is concentrated in the less abundant, or rare, fractions of the community, i.e. the "long tail" of the abundance distribution. The relationship between microbial community structure and activity, specifically the role of rare microbes, and its connection to ecosystem function, is not fully understood. We analyzed 12.3 million metagenomic and metatranscriptomic sequence assemblies and their genes from environmental, human, and engineered microbiomes, and show that microbial activity is dominated by rare microbes ( $96 \%$ of total activity) across all measured biomes. Further, rare microbial activity was comprised of traits that are fundamental to ecosystem and organismal health, e.g. biogeochemical cycling and infectious disease. The activity of rare microbes was also tightly coupled to temperature, revealing a link between basic biological processes, e.g. reaction rates, and community activity. Our study provides a broadly applicable and predictable paradigm that implicates rare microbes as the main microbial drivers of ecosystem function and organismal health.

\section{Background}

Members of the rare biosphere have been recognized as important drivers of many key ecosystem functions ${ }^{1-6}$. Rare microbes may control ecosystems as keystone members, i.e. community members that the whole ecosystem depends. For example, marine rhizobia are rare, but control the input of bioavailable nitrogen via $\mathrm{N}_{2}$ fixation ${ }^{7}$. Also, rare microbes may be disproportionately active relative to their abundance, e.g. the rarest detectable taxon by cell count in Lake Cadagno, Switzerland, an oligotrophic lake, was discovered to contribute to $>40 \%$ and $>70 \%$ of the total ammonium and carbon uptake ${ }^{8}$, respectively. However, these instances do not demonstrate the influence of rare microbes on total community activity. Sequencing of RNA 
transcripts and DNA of single marker genes have been employed to understand the influence of rare microbes on community activity. These methods have revealed that rare microbes are potentially more active than abundant ones ${ }^{9-11}$, but can suffer from over extrapolations of genome wide function and activity from a single gene ${ }^{12}$. Specifically, ratios of rRNA gene transcripts and rRNA gene quantities have been shown to be poor indicators of cell wide activity $^{13}$, e.g. cyanobacteria can have elevated levels of rRNA in dormant cells relative to vegetative cells ${ }^{14}$.

To better understand the influence of rare microbes on community activity we employed a systems based approach to examine the molecular activity of the genomic content of microbiomes. We de novo assembled environmental DNA and RNA shotgun sequences from the genomic and transcriptomic reservoir of the global microbiome. Broadly, data are sourced from publicly available and novel environmental, host-associated, and human-engineered shotgun sequenced communities. Samples encompass the ocean, Amazon River ${ }^{15}$ and its plume into the ocean $^{16,17}$, the human gut ${ }^{18}$, permafrost soil layers ${ }^{19}$, a thermokarst bog $^{19}$, and human-engineered biogas plants ${ }^{20,21}$. Ocean samples span: the sunlit epipelagic ${ }^{22-28}(0-200 \mathrm{~m})$, the dimly lit mesopelagic (200 - 1,000 m), dark bathypelagic $(1,000-4,000 \mathrm{~m})$, the benthic zone (near seafloor), and hydrothermal vent plumes ${ }^{29,30}$ (Table S1). Our analysis uses database independent de novo high resolution 99\% average nucleotide (ANI) contiguous assemblies that captures the entire genomic repertoire from all domains of life. Using these assemblies, we examined the activities of rare and abundant microbes and their functional traits across many disparate environments. 


\section{Results/Discussion}

Microbial activity is consistently and commonly dominated by rare microbes. Relating community structure as a function of community activity in rare sequence assemblies across disparate environments consistently showed that total RNA expression of rare microbes was many folds higher than the total RNA expression of abundant microbes (Figs. 1, S1 - S3). Approximately $96 \%$ of all microbial activity was contributed by rare microbes (Table S2). In $>90 \%$ of samples, $>90 \%$ of total community activity was in the rare fraction (Fig. S4). Rare microbes were defined as those with sequence assemblies that are in the "tail" of the DNA rank abundance curve ${ }^{31}$, in this study $>1000$ th rank or $\sim 0.005 \%$ by relative abundance (Fig. S5A). Microbial activity was also dominated by rare microbes using assembly independent kmer counting (Fig. S3A), indicating that our finding is not a result of sequence assembly bias. Further, rare microbial expression was also highly overrepresented at the gene level (Fig. S3B), i.e. open reading frames (ORFs), and an approximate functional level based on clustering of ORFs at $60 \%$ amino acid identity (Fig. S3C). Sense, or coding strand, RNA transcript expression of ORFs was higher than antisense expression for rare microbes (Mann-Whitney $U$ test; $P<2.2$ $10^{-16}$ ) that were from samples prepared with methods that retained strand orientation (Figs. 2D and S6). Sense mRNAs are transcripts that are meant for downstream translation into protein, whereas antisense transcripts primarily act as post-transcriptional regulators by directly binding to sense transcripts ${ }^{32}$. Higher sense transcription indicates that most microbial transcription is ultimately meant for protein translation. This pattern is consistent across all sampled environments and lifestyles, e.g. free-living or attached (Fig. 2E). Further, our analysis captures the entire genomic representation of microbial communities and is not limited to single genes or processes. 
Next, we examined the structure of microbial abundance and activity across environments. Samples clustered by environment across DNA, RNA, and specific activity ${ }^{10}$ (RNA:DNA) distributions (Fig. S7). Further, the rank abundance distributions, i.e. measures of biodiversity, from all environments using sequence assemblies follow a highly skewed curve, as has been widely reported for microbial communities using single-marker genes ${ }^{5,31,33}$ (Fig. S5A). This demonstrates that highly skewed rank abundance curves even at a genomic level are a consistent feature of microbial communities regardless of environment. Highly skewed rank abundance curves suggest that there are dominant genotypes in microbial populations, despite potentially high recombination rates ${ }^{34}$. The prevalence of dominant genotypes indicate that ecological pressures, e.g. nutrient limitation and predation, can select for specific "winning" genotypes among highly related microbial populations. RNA activity patterns were similarly skewed by rank expression, demonstrating that microbial activity is similarly dominated by a small number of overrepresented assembled sequences (Fig. S5B). Community activity was also more skewed toward a few assemblies, as indicated by lower Pielou's evenness $\left(J^{\prime}\right)$ compared to community structure $J^{\prime}$ (Fig. S8; Mann-Whitney $U$ test; $P<2.2 \times 10^{-16}$ ). The rank abundance and rank activity curves reflect that not only do a small number of microbes dominate community structure, but fewer types, relative to community structure, also dominate community activity.

The extent that rare microbes contributed to activity varied within and between environments (Fig. 2A). This pattern was driven by temperature variation, as total activity of rare microbes decreased with increasing temperature (Fig. 2B; Spearman's $\rho=-0.42 ; P=3.979 \times 10^{-7}$ ). The degree that community activity was dominated by fewer microbes, i.e. activity dominance, alternatively increased with increasing temperature (Fig. 2C; Spearman's $\rho=0.7 ; P<2.2 \times 10^{-}$ 
$\left.{ }^{16}\right)$. Temperature is a first order determinant of chemical reaction kinetics, and, therefore, biochemical processes ${ }^{35}$. Higher temperatures induce higher metabolic rates that ultimately mediate biological activities ${ }^{36}$. This positive relationship between temperature and biological process rates has been implicated in controlling many ecological ${ }^{37-39}$ and evolutionary ${ }^{40,41}$ patterns. Our observations show that temperature is also a major mediator of the structure of microbial community activity.

We explored the functional contribution of rare microbes to community activity across all environments. Functional traits were examined that were more than two fold overexpressed in the rare fraction relative to the abundant fraction (Mann-Whitney $U$ test; $P<3.9 \times 10^{-71}$ ). Rare ORFs were enriched in the activity of functional traits that have a direct influence on total ecosystem function (Fig. 3), e.g. energy production and conversion, carbohydrate transport and metabolism, coenzyme transport and metabolism, inorganic ion transport and metabolism, nucleotide transport and metabolism, amino acid transport and metabolism, lipid transport and metabolism. Rare ORFs were also enriched for functional traits involved with cell motility, coinciding with the observation that rare microbes tend toward chemotactic lifestyles ${ }^{42-44}$ (Fig. 3A). Other processes linked to growth were also overrepresented in rare ORFs, including cell growth and death, cell cycle control, cell division, chromosome partitioning, cell wall/membrane/envelope biogenesis, and translation (Fig. 3AB). This suggests that rare microbes have higher growth rates, as well as the aforementioned higher metabolic activity. Although higher growth rates should result in higher abundance, rare microbes were enriched in defense mechanisms and xenobiotic degradation, suggesting that they are subject to higher pressures of viral predation, grazing, host-defense, and allelopathy (Fig. 3B). Rare ORFs were 
also enriched in infectious disease categories, implicating rare microbes in animal and human disease. Finally, many of the genes most expressed by rare microbes were directly involved in major biogeochemical processes, such as photosynthesis, $\mathrm{N}_{2}$ fixation, and ammonia oxidation (Fig. 3C). For example, a rare Candidatus Atelocyanobacterium thalassa ${ }^{45}$ (unicellular cyanobacteria group A member) had a nifH (iron binding component of nitrogenase) with the highest annotatable contribution to activity in the epipelagic South Atlantic Ocean (Table S3). Other relevant biochemical processes include ammonium, phosphate, and energy driven carbohydrate $\mathrm{ABC}$ transport. The influence of rare microbes in mediating important ecosystem processes highlights their role as keystone members of ecosystems.

Deciphering the role of rare microbes in microbial communities is at the core of understanding the influence that microbes have on ecosystem function. We demonstrate that rare microbes are not only more active, but dominate microbial community activity in many different environments. This pattern is consistent across many disparate environments, ranging from the human gut to human engineered biogas plants to hydrothermal vents. The contribution of rare microbes to community activity varies across environments and is strongly influenced by temperature, implying that fundamental biological processes, e.g. reaction rates, control community activity structure. Rare microbes were more involved than abundant microbes in important ecosystem processes, e.g. energy transformation and biogeochemical cycling. Rare microbial activity was also enriched in genes related to infectious disease, underscoring the detrimental impacts of rare microbes on animal and human health. Our observations indicate that the dominance of rare microbial activity is a conserved trait for all of Earth's biomes. 


\section{Figures}
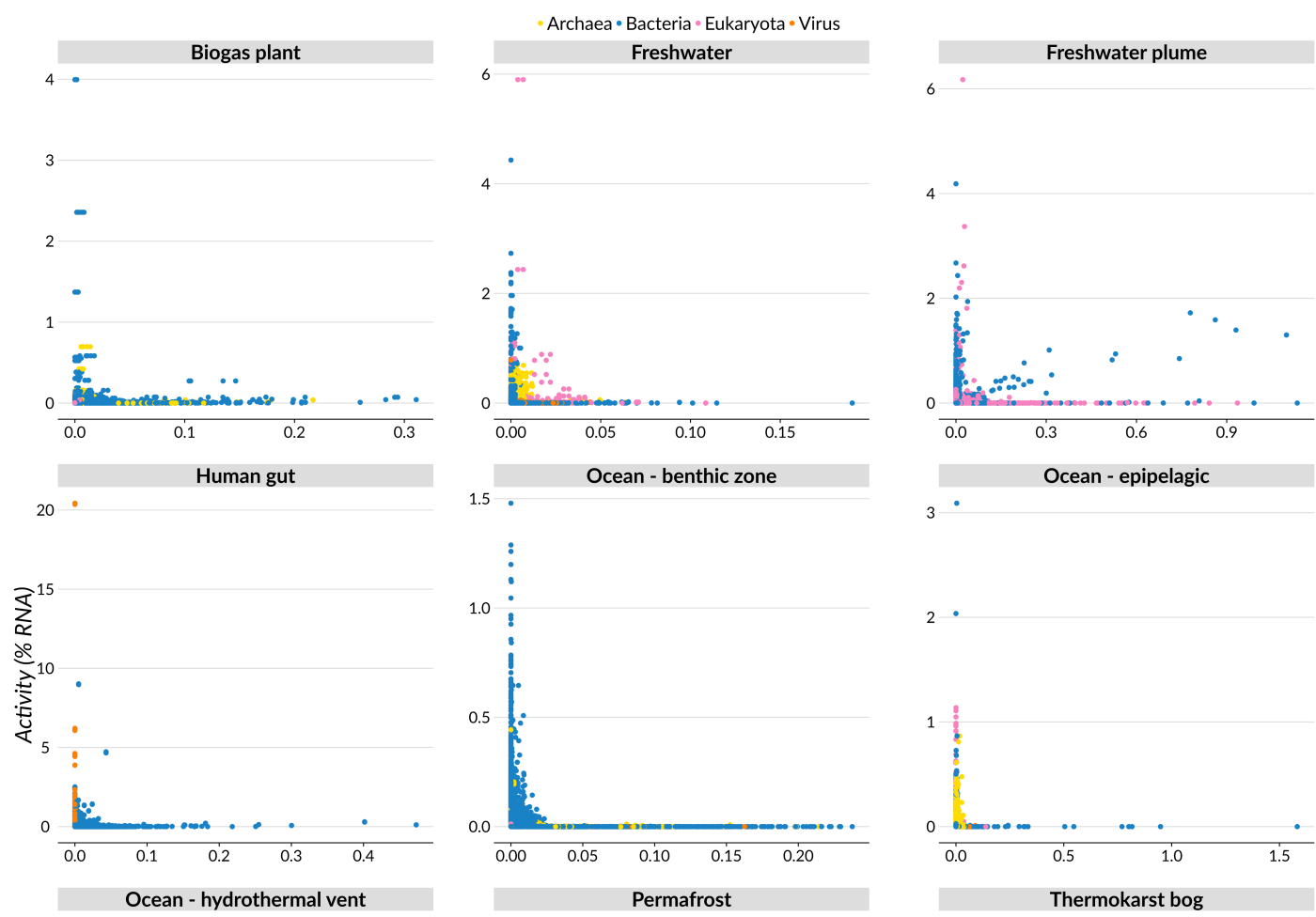

3
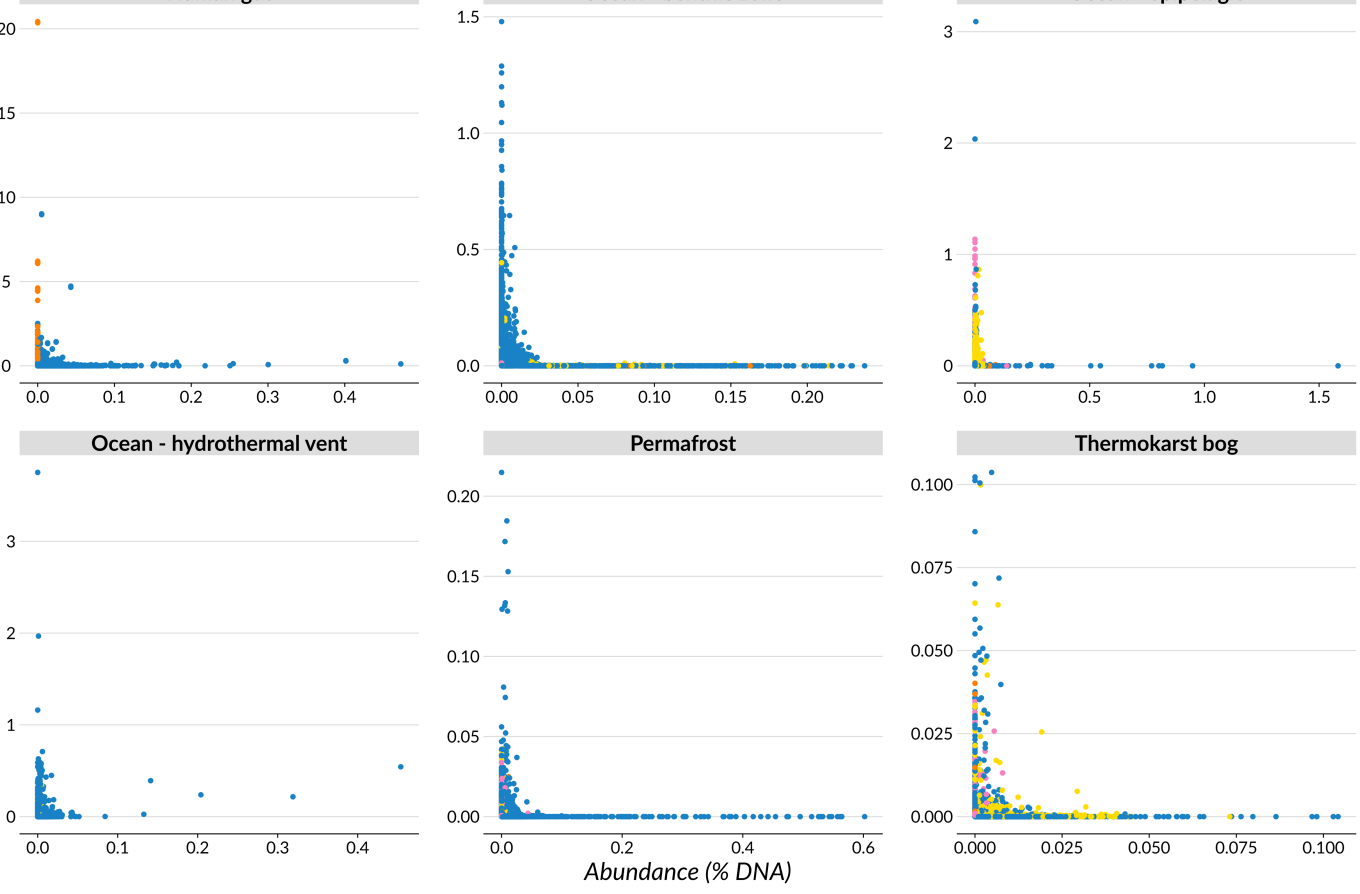

Figure 1. Relationships between community structure and community activity of highly resolved sequence assemblies across disparate environments. Each point represents a sequence assembly and its relative contribution to community structure and activity. Environments are biogas plants, freshwater, freshwater plume into the ocean, the human gut, ocean (epipelagic, benthic zone, hydrothermal vents), permafrost, and thermokarst bog. Community structure is expressed as relative frequencies of DNA and community activity as relative frequencies of RNA of samples sequenced with Illumina platforms. All frequencies were adjusted to sequence assembly length and subsampled to account for uneven sequencing effort. Points are colored by RefSeq lowest common ancestor (LCA) taxonomy at the domain rank. 


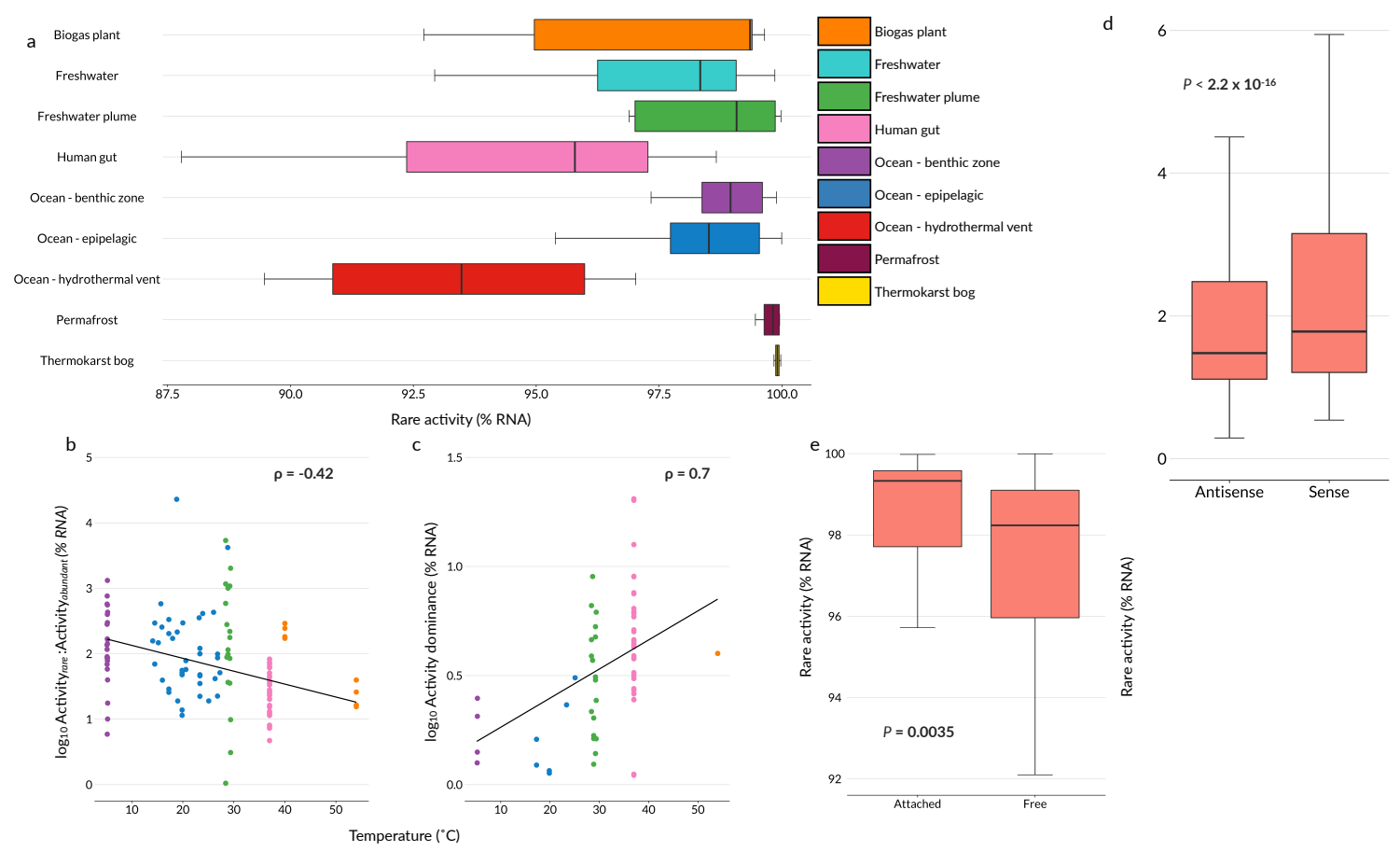

Figure 2. Patterns of rare high-resolution sequence assembly activity of Illumina sequenced samples across different environments, lifestyles, and temperatures. a) Box and whisker plots of rare assembly activity across different environments. Mann-Whitney $U$ test; $P<2.2 \mathrm{x}$ $10^{-16}$. b) Rare microbial activity expressed as a ratio of $\log _{10}$ rare:abundant $\%$ RNA as a function of temperature. Linear regression plotted with Spearman's $\rho=-0.42$ and $\mathrm{P}=1.701 \times 10^{-7}$. c) Activity dominance expressed as Berger-Parker dominance of \% RNA as a function of temperature. Linear regression plotted with Spearman's $\rho=-0.7$ and $P<2.2 \times 10^{-6}$. d) Box and whisker plots of rare antisense and sense microbial activity. e) Box and whisker plots of rare microbial activity in attached and free, i.e. planktonic, lifestyles. Samples with microbes with attached lifestyles were considered: sediment samples, i.e. permafrost, thermokarst bog, and aquatic (freshwater and marine) samples collected on filters $>0.8 \mu \mathrm{m}$. Samples with microbes with free lifestyles were considered: the human gut and aquatic (freshwater and marine) samples collected on filters $0.1-3.0 \mu \mathrm{m}$. 

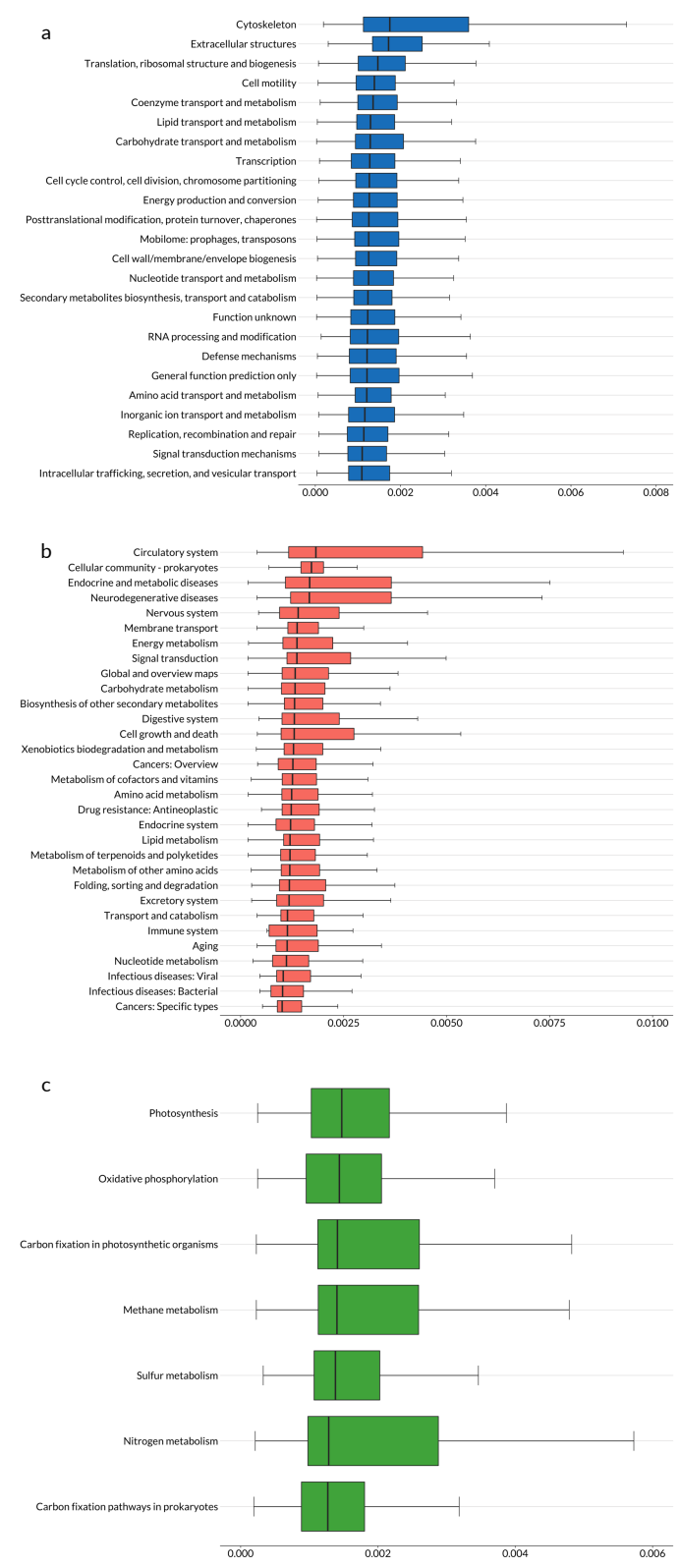

Figure 3. Functional traits of Illumina sequenced rare microbial activity enriched in rare fraction relative to the abundant fraction. Overexpression of traits in the rare fraction was determined by selecting ORFs with $>2 \mathrm{X}$ activity in the rare fraction that had a Mann-Whitney $\mathrm{U}$ with $P<1 \times 10^{-70}$ a) Box and whisker plots of rare overexpressed NCBI Clusters of Orthologous Groups (COGs) functional categories. c) Box and whisker plots of KEGG BRITE categories overexpressed in the rare fraction. d) Box and whisker plots of KEGG BRITE "Energy metabolism" subcategories overexpressed in the rare fraction. Box and whisker plots are sorted by median and exclude ORFs where \% RNA was 0. 


\section{Methods}

\section{Sargasso Sea sample collection and sequencing}

Four samples were collected from the end of spring (3/24/2010 and 3/26/2010) and summer (8/20/2010 and 8/22/2010) from the Bermuda Atlantic Time Series station (BATS, 31 ${ }^{\circ} 29^{\prime} 46^{\prime \prime}$

$\left.\mathrm{N}, 63^{\circ} 59^{\prime} 52^{\prime \prime} \mathrm{W}\right) .5$ - $20 \mathrm{~L}$ were collected from a depth of $50 \mathrm{~m}$ and immediately amended with an equal volume of RNAlater once on board ship. The RNAlater amended samples were sequentially filtered through a glass fiber $0.8 \mu \mathrm{m} \mathrm{GF} / \mathrm{F}$ filter (Whatman) and finally onto a 0.22 $\mu \mathrm{m}$ Durapore (Millipore) filter within one hour of collection and stored at $-80{ }^{\circ} \mathrm{C}$. DNA and RNA were extracted as previously described in Campbell et al. 200946. DNA was fragmented with a Covaris S2 with the recommended parameters to generate $300 \mathrm{bp}$ inserts prior to metagenome library preparation using an Encore NGS Library System I (NuGEN). RNA was purified from DNA as described previously ${ }^{46}$ and approximately $1 \mu \mathrm{g}$ from spring samples were rRNA subtracted using the MICROBExpress Bacterial mRNA Enrichment Kit (Ambion). All RNAs were reverse transcribed following the manufacturer's instructions for cDNA synthesis and metatranscriptome libraries prepared with an Ovation RNA-Seq System (NuGEN) kit. Libraries were sequenced via a paired end 2 x 100 bp strategy using an Illumina HiSeq 2000. Libraries from DNA extracted from 3/24/2010 and 8/20/2010 samples were also sequenced on the Roche 454 GS FLX+ platform and using circular consensus sequencing on a PacBio RS.

\section{San Pedro Ocean Time-series (SPOT) benthic zone sample collection and extraction}

Seawater samples $(1-20$ L) were collected approximately monthly over $\sim 2.5$ years $(\mathrm{n}=32)$ from near the ocean floor $(890 \mathrm{~m})$ in the Southern California Bight at the SPOT station $\left(33^{\circ}\right.$ $33^{\prime} \mathrm{N}, 118^{\circ} 24^{\prime} \mathrm{W}$ ) aboard the $R / V$ Yellowfin. Samples were serially filtered through a nylon mesh 
$(80 \mu \mathrm{m})$, Acrodisc (Pall) glass fiber filter $(\sim 1 \mu \mathrm{m})$, and terminally with a Sterivex-GP (Millipore) polyethersulfone filter $(0.22 \mu \mathrm{m})$. Acrodisc and Sterivex filters were preserved with $250 \mu 1$ and $500 \mu \mathrm{l}$ of RNAlater, respectively, and subsequently incubated at ambient temperature for $2 \mathrm{~min}$. Samples were immediately frozen in $\mathrm{LN}_{2}$ and stored at $-80^{\circ} \mathrm{C}$. The $0.22 \mu \mathrm{m}-1 \mu \mathrm{m}$ fraction was selected for sequencing and DNA was extracted from each Sterivex cartridge using a modified AllPrep DNA/RNA (Qiagen) kit. RNAlater was sparged from each Sterivex using a syringe. High salt concentrations can lower DNA yields with the AllPrep DNA/RNA kit. To desalt RNAlater while maximizing nucleic acid concentrations we used an Amicon Ultra 3k. Briefly, $500 \mu \mathrm{l}$ RNAlater (Ambion) was transferred to an Amicon Ultra 3k $0.5 \mathrm{ml}$ spin concentrator and centrifuged at $14,000 \mathrm{~g}$ for $30 \mathrm{~min}$ at $4^{\circ} \mathrm{C}$. This was repeated with any remaining unconcentrated RNAlater. $400 \mu 1$ of $4^{\circ} \mathrm{C}$ nuclease free water was added directly to the concentrator and concentrate to desalt and centrifuged at $14,000 \mathrm{~g}$ for $1 \mathrm{hr}$ at $4{ }^{\circ} \mathrm{C} 2 \mathrm{ml}$ of $65^{\circ} \mathrm{C} \mathrm{RLT}+$ lysis buffer and $20 \mu$ l beta-mercaptoethanol were added to the previously desalted and concentrated RNAlater. The Sterivex cartridge was sealed with luer lock caps and vortexed for $5 \mathrm{~s}$, flipped and vortexed again for $5 \mathrm{~s}$. The Sterivex cartridge was horizontally rotated for $15 \mathrm{~min}$ at $65^{\circ} \mathrm{C}$. The lysate was removed, and the Sterivex filter was lysed again with $1 \mathrm{ml} \mathrm{RLT}+$ and $10 \mu \mathrm{l}$ betamercaptoethanol. The lysates were combined and DNA was purified following the manufacturer's protocol and finally eluted with buffer AE. RNA was purified from the DNA purification flow through following the manufacturer's instructions with a 30 min on-column DNAse step and a final elution with $50 \mu 1$ nuclease free water. $1 \mu 1$ of RiboGuard RNase Inhibitor (Epicentre) was added to the eluted RNA to protect against ribonucleases. RNA quantities were determined using a Qubit RNA High Sensitivity (ThermoFisher) kit. 


\section{SPOT benthic zone metagenome library construction and sequencing}

DNA concentrations were determined for each sample using a Qubit High Sensitivity DNA assay (Invitrogen) and diluted to $10 \mathrm{ng}$. Samples were amended with the DNA of 4 exotic genomes from American Type Culture Collection with a range of 34.5 - $59.9 \% \mathrm{G}+\mathrm{C}$ content (Table S4). Each genome was added in 2 fold increasing concentrations at $\sim 1 \%$ of the total DNA concentration of each sample. Metagenomes were prepared from each amended sample using a Covaris S2 (130 $\mu \mathrm{l})$ with parameters: duty cycle (10\%), intensity (5) cycles/burst (200), time (60 s). Insert and dual indexed libraries were prepared using a modified NEBNext Ultra DNA II dual indexing kit (New England Biolabs) without size selection. The protocol was modified to control for chimeric PCR amplicons and reduce overamplification biases. Adaptor ligated and end repaired fragments were amplified with 0.1X of 10,000X SYBR Green I (Invitrogen). Amplification was monitored on a CFX96 real-time PCR machine (BioRad) and stopped in the exponential phase to avoid overamplification. The reaction was held at $98^{\circ} \mathrm{C}$ for $30 \mathrm{~s}$ followed by 6 cycles of amplification at $98^{\circ} \mathrm{C}$ for $10 \mathrm{~s}, 65^{\circ} \mathrm{C}$ for $5 \mathrm{~min}$, and a final extension of $30 \mathrm{~min}$. Extension times were increased to reduce chimeric amplicons. Libraries were subsequently $2 \mathrm{x}$ $250 \mathrm{bp}$ paired end sequenced on an Illumina HiSeq 2500.

\section{SPOT benthic zone metatranscriptome library construction and sequencing}

40 ng of RNA was amended with $8 \mu$ of a 10,000X dilution of External RNA Control Consortium $^{47}$ (ERCC) Spike-In Mix 1 (Ambion). The ERCC mix consists of 92 transcripts ranging from 250 to 2,000 nt in length with a large dynamic fold range. The transcripts are mostly novel synthesized sequences, but do contain Bacillus subtilis transcripts. The added genome and ERCC controls are sequencing controls used to verify that our sample preparation 
and bioinformatic protocols are accurate and reproducible. The amended RNA samples were rRNA depleted using a RiboZero Bacteria rRNA removal kit. RNA was fragmented using a Covaris S2 targeting 600 bp: sample volume $(130 \mu \mathrm{l})$, peak incident power $(50 \mathrm{~W})$, duty factor (20\%), cycles per burst $(200)$, treatment time $(60 \mathrm{~s})$, temperature $\left(7^{\circ} \mathrm{C}\right)$. Strand specific cDNA was generated using random priming without size selection from a NEBNext Ultra RNA directional kit. Illumina libraries were constructed from the resulting cDNA using a modified NEBNext DNA Ultra II dual indexing kit. The protocol was modified to use RT-PCR to control for overamplification and longer extension times to control for chimeric amplicons as previously described (Chapter 1), and resulted in 12 cycles of amplification. Metagenomic and metatranscriptomic libraries were sequenced 2 x 250 bp using an Illumina HiSeq 2500.

\section{Sequence and metadata retrieval}

Raw metagenomic and metatranscriptomic reads from Illumina, 454, Sanger, and PacBio sequencing platforms from 504 sequence libraries covering a number of disparate environments were downloaded for each publicly available dataset (Table S1). Ocean samples encompass the epipelagic, mesopelagic, bathypelagic, the benthic zone, and hydrothermal vent plumes, ranging in depths 0 - 4,946 m. Epipelagic samples include coastal and open ocean sites. Other samples come from the Amazon River and its plume into the Atlantic Ocean, active and frozen permafrost, a thermokarst bog, human guts, and biogas plants. Data were retrieved from iMicrobe, NCBI SRA, and ENA (Table S1). Metagenome and metatranscriptome sample pairings and sample metadata (e.g. temperature and environment) were determined using sequence database metadata or directly from the source publications. Human gut sample 
temperatures were not publicly available and were inferred to be $37^{\circ} \mathrm{C}$ based on the typical human body temperature ${ }^{48}$.

\section{Sequence quality control and assembly}

The majority of samples were sequenced using Illumina based flow cell technologies (Table S1). Illumina reads were adapter and quality trimmed in one pass to retain the largest regions with Q $>25\left(\right.$ BBMap $^{49}$ v36.19; bbduk.sh qtrim $=\mathrm{rl}$ trimq $=25 \mathrm{ktrim}=\mathrm{r} \mathrm{k}=25 \mathrm{mink}=11$ hdist $=1$ ref=truseq.fa.gz). Reads from long read sequencing platforms, Roche 454, Sanger, and Pacbio RS, were similarly trimmed to $\mathrm{Q}>20$ (BBMap v36.19; bbduk.sh qtrim=rl trimq=20). Genome and ERCC controls were removed from each SPOT benthic zone sample prior to assembly and mapping. Reads were mapped to the added genome and ERCC controls at $95 \%$ identity and the unmapped reads and their paired end mates were retained for downstream assembly and mapping $\left(\mathrm{BBMap}^{49} \mathrm{v} 36.19\right.$; bbmap.sh idfilter=0.95). Each metagenome and metatranscriptome from each sample was individually assembled de novo using MEGAHIT ${ }^{50}$ in paired end mode if sequence libraries were paired end sequenced. MEGAHIT was run to ensure that no bubbles were merged that were $<99 \%$ to ensure that only highly-resolved assemblies were generated (MEGAHIT v1.0.6; megahit --merge-level 20, 0.99 --k-min 21 --k-max 255 --k-step 6). All assemblies were combined and dereplicated using a semi-global alignment method that merged together assemblies that were totally contained and >99\% similar (BBMap v36.19; dedupe.sh minidentity $=0.99)$. Assemblies $<1 \mathrm{~kb}$ were discarded. Processing resulted in $12,338,658$ assemblies, comprised of $25.48 \mathrm{Gbp}$. 


\section{Annotation}

Open reading frames (ORFs) were predicted (ORFfinder ${ }^{51}$-s 1 -n T) and ORFs $>200$ amino acids were retained. The resulting ORFs were searched against the KEGG reference database (DIAMOND $^{52}$; diamond blastp -e 1e-5 --sensitive), KEGG modules and pathways were retrieved using the KEGG API. Only the best hits with E-value $<1 \times 10^{-10}$ were assigned. ORFs were also searched against the complete non-redundant NCBI RefSeq Release $83^{53}$ protein database (DIAMOND; diamond blastp --top 5 -e 1e-5) and hits with E-value $<1 \times 10^{-10}$ were retained. A best hit was determined by sorting by E-value, bit score, and percent identity. Taxonomy was assigned for each sequence assembly using a lowest common ancestor (LCA) approach. RefSeq hits for each ORF that were within $5 \%$ of the bit score of the best hit were retained. The remaining hits were used to assign a LCA taxonomy for each assembly.

\section{Metagenome and metatranscriptome mapping and counting}

Mapping and counting were performed within each sequencing platform where both metagenomes and metatranscriptomes were sequenced, i.e. Roche 454 and Illumina platforms. Small subunit (16S and 18S) and large subunit (5S, 5.8S, 23S, and 28S) rRNAs were removed from each read set prior to mapping reads from each environment to each dereplicated set of assemblies (SortMeRNA ${ }^{54}$ v2.1; sortmerna --paired_out --fastx --ref silva-bac-16s-id90.fasta silva-arc-16s-id95.fasta silva-euk-18s-id95.fasta rfam-5s-database-id98.fasta rfam-5.8s-databaseid98.fasta silva-arc-23s-id98.fasta silva-bac-23s-id98.fasta silva-euk-28s-id98.fasta). The resulting rRNA filtered reads from each library were mapped to the dereplicated set of assemblies. Filtered reads were mapped to retain all sites with the highest score, ie. the assemblies that are the best matches, and >99\% identity (BBMap v36.19; bbmap.sh 
ambiguous $=$ all maxsites $=1000000000$ maxsites $2=1000000000 \mathrm{sssr}=1.0$ secondary $=\mathrm{t}$ minid $=0.98$ idfilter=0.99).

Mapped counts were determined for each sample and all mapped sites with a minimum read length of $50 \mathrm{bp}$ were considered (featureCounts ${ }^{55}$ v1.5.3; featureCounts -f -O -M --minOverlap 50 -s 0 ). ORF expression and abundance were similarly counted by using the previously mapped reads and ORF start and stop locations for counting (featureCounts v1.5.3; featureCounts -f -O M --minOverlap 50 -s 0). Strand specific, i.e. sense and antisense, counts were determined for RNA libraries prepared to retain strand information (Table S1) using featureCounts v1.5.3 in forward (featureCounts v1.5.3; featureCounts -f -O -M --minOverlap 50 -s 1) and reverse (featureCounts v1.5.3; featureCounts -f -O -M --minOverlap 50 -s 2) strand counting modes. All strand specific libraries were prepared using methods that result in sequencing reads that are the reverse complement of the transcribed RNA sequence. Accordingly, the reverse counts were considered as the sense counts and the forward counts as the antisense counts. Next, assemblies that matched to the Centrifuge ${ }^{56}$ NCBI nucleotide index ${ }^{56}(12 / 06 / 2016)$ and matches to human sequences $>90 \%$ assemblies coverage were removed (Centrifuge v1.0.3-beta ${ }^{56}$; default parameters). Assemblies that matched to the genome, ERCC, and PhiX 174 controls were also removed (NCBI BLAST $+{ }^{57}$ v2.6.0; blastn -evalue 0).

Prior to counting, assemblies matching mapped counts were divided by assembly length or ORF length to account for higher recruitment of longer assemblies or ORFs. Assemblies and unstranded ORF length adjusted counts were subsampled, i.e. rarefied, to the length adjusted counts of the smallest sample to account for uneven sequencing coverage. Stranded forward and 
reverse length adjusted counts were subsampled to the total forward and reverse length adjusted counts of the smallest sample. Subsampling was done separately for Illumina and 454 sequences to maintain high counts for Illumina samples because the 454 based sequencing effort was much lower than the Illumina based sequencing effort. Length adjusted and subsampled counts for each assembly or ORF were normalized to the total length normalized count of each sample to obtain a relative count, e.g. relative DNA abundance or relative RNA expression. Pairings between RNA and DNA counts were determined from sample database metadata or from the sample publications. In some cases, there were multiple pairings with a sample. Some samples had $>1$ DNA or RNA library sequenced. For example, if a sample has 2 DNA and 2 RNA sequence libraries, there are 4 possible DNA and RNA pairs. All data manipulations were done using Python ${ }^{58}$ and Pandas ${ }^{59}$. Specific activity was determined as a ratio by dividing relative RNA and relative DNA counts ${ }^{10}$.

\section{SPOT benthic zone metagenomic and metatranscriptomic controls}

Genome (DNA) and ERCC (RNA) controls were analyzed using the same mapping and counting protocols that we used to quantify abundance and expression. Mapping and counting were performed exactly as for mapping and counting of all Illumina sequences from all environments. Trimmed and rRNA filtered metagenomic and metatranscriptomic from the SPOT benthic zone samples were mapped to the genome and ERCC control sequences (BBMap v36.19; bbmap.sh ambiguous $=$ all maxsites $=1000000000$ maxsites $2=1000000000 \mathrm{sssr}=1.0$ secondary $=\mathrm{t}$ minid $=0.98$ idfilter=0.99). Counts (featureCounts v1.5.3; featureCounts -f -O -M --minOverlap 50 -s 0) were transformed into relative counts by length dividing and normalizing to the total mappings from each sample. Performance was quantified by plotting against the expected genome and ERCC 
control concentrations. Both genome and ERCC controls had high agreement between input quantities and measurements $\left(\mathrm{R}^{2}>0.99\right)$ using the afforementioned protocols (Fig. S9).

\section{Approximate functional clusters}

Functional clusters were generated by clustering ORFs based on percent identity. ORFs longer than 200 amino acids were grouped into protein clusters with $>60 \%$ identity (cd-hit ${ }^{60}$ v4.6; cd-hit -c 0.6 -n 4). Relative counts for clusters were determined by summing the length adjusted subsampled normalized counts of each ORF contained within a cluster.

\section{Assembly free kmer based analysis}

Two samples were randomly selected from each environment for verification of abundance and activity relationships using an assembly free kmer counting. Counts of all canonical 31 letter kmers were generated for each sample (BBMap v36.19; kmercountexact.sh k=31). Kmers of 31 letters were chosen because they have been shown to be able to distinguish approximate microbial species ${ }^{61}$. Kmers with low complexity (Shannon entropy $\leq 1.84$ ) were removed to increase the likelihood of capturing kmers that can separate microbes at the species-level.

\section{Diversity metrics and statistics}

Diversity metrics were calculated using relative counts for metagenomes and metatranscriptomes. Pielou's evenness $\left(J^{\prime}\right)$ and Berger-Parker dominance were calculated using scikit-bio. Linear regressions and nonparametric statistics were calculated using Spearman's $\rho$ and the Mann-Whitney $\mathrm{U}$ test from the $\mathrm{R}^{62}$ stats package. Non-metric dimensional scaling plots 
(NMDS) were generated using the vegan ${ }^{63} \mathrm{R}$ package and Bray-Curtis dissimilarities (vegan v2.4-4; metamds (distance="bray").

\section{Acknowledgements}

We would like to acknowledge the Wrigley Institute for Environmental Studies, the crew of the $R / V$ Yellowfin, and Troy Gunderson for logistical support of SPOT samples. We thank Jed Fuhrman and his lab, including Erin Fichot, Catherine Garcia, David Needham, Alma Parada, Ella Sieradzki, Jacob Cram, and Vicki Trinh. We also thank Megan Hall for helpful comments on drafts of the manuscript. We thank the scientists who deposited their sequences in publicly accessible databases. This work was funded by the Wrigley Graduate Summer Fellowship Program and National Science Foundations awards 12053, 0824981, and 0939564 to J.F.H., and 0825468 and 1261359 to B.J.C.

\section{Contributions}

R.S., B.J.C, and J.F.H designed the study for epipelagic and benthic marine environments. R.S. expanded the study to other environments. R.S. prepared SPOT benthic zone metagenomes and metatranscriptomes. B.J.C prepared Sargasso Sea metagenomes and metatranscriptomes. R.S. analyzed the data. R.S wrote the paper with input from B.J.C and J.F.H. All authors read and approved the manuscript.

\section{Competing interests}

The authors declare no competing interests. 
bioRxiv preprint doi: https://doi.org/10.1101/636373; this version posted May 16, 2019. The copyright holder for this preprint (which was not certified by peer review) is the author/funder, who has granted bioRxiv a license to display the preprint in perpetuity. It is made available under aCC-BY-NC-ND 4.0 International license.

\section{Data availability}

Sargasso Sea sequences are deposited under NCBI SRA BioProject PRJNA242360. SPOT sequences are currently being deposited at NCBI. 


\section{References}

1. Graham, E. B. et al. Microbes as Engines of Ecosystem Function: When Does Community Structure Enhance Predictions of Ecosystem Processes? Front. Microbiol. 7, 214 (2016).

2. Naeem, S. \& Li, S. Biodiversity enhances ecosystem reliability. Nature 390, 507-509 (1997).

3. Carney, K. M. \& Matson, P. A. Plant Communities, Soil Microorganisms, and Soil Carbon Cycling: Does Altering the World Belowground Matter to Ecosystem Functioning? Ecosystems 8, 928-940 (2005).

4. Nelson, M. B., Martiny, A. C. \& Martiny, J. B. H. Global biogeography of microbial nitrogen-cycling traits in soil. Proc. Natl. Acad. Sci. U. S. A. 113, 8033-8040 (2016).

5. Fuhrman, J. A. Microbial community structure and its functional implications. Nature 459, 193-199 (2009).

6. Anantharaman, K. et al. Thousands of microbial genomes shed light on interconnected biogeochemical processes in an aquifer system. Nat. Commun. 7, 13219 (2016).

7. Sohm, J. A., Webb, E. A. \& Capone, D. G. Emerging patterns of marine nitrogen fixation. Nat. Rev. Microbiol. 9, 499-508 (2011).

8. Musat, N. et al. A single-cell view on the ecophysiology of anaerobic phototrophic bacteria. Proc. Natl. Acad. Sci. U. S. A. 105, 17861-17866 (2008).

9. Campbell, B. J., Yu, L., Heidelberg, J. F. \& Kirchman, D. L. Activity of abundant and rare bacteria in a coastal ocean. Proc. Natl. Acad. Sci. U. S. A. 108, 12776-12781 (2011).

10. Hunt, D. E. et al. Relationship between abundance and specific activity of bacterioplankton in open ocean surface waters. Appl. Environ. Microbiol. 79, 177-184 (2013).

11. Kamke, J., Taylor, M. W. \& Schmitt, S. Activity profiles for marine sponge-associated 
bacteria obtained by $16 \mathrm{~S}$ rRNA vs $16 \mathrm{~S}$ rRNA gene comparisons. ISME J. 4, 498-508 (2010).

12. Blazewicz, S. J., Barnard, R. L., Daly, R. A. \& Firestone, M. K. Evaluating rRNA as an indicator of microbial activity in environmental communities: limitations and uses. ISME J. 7, 2061-2068 (2013).

13. Cottrell, M. T. \& Kirchman, D. L. Transcriptional Control in Marine Copiotrophic and Oligotrophic Bacteria with Streamlined Genomes. Appl. Environ. Microbiol. 82, 6010-6018 (2016).

14. Sukenik, A., Kaplan-Levy, R. N., Welch, J. M. \& Post, A. F. Massive multiplication of genome and ribosomes in dormant cells (akinetes) of Aphanizomenon ovalisporum (Cyanobacteria). ISME J. 6, 670-679 (2012).

15. Satinsky, B. M. et al. Metagenomic and metatranscriptomic inventories of the lower Amazon River, May 2011. Microbiome 3, 39 (2015).

16. Satinsky, B. M. et al. The Amazon continuum dataset: quantitative metagenomic and metatranscriptomic inventories of the Amazon River plume, June 2010. Microbiome 2, 17 (2014).

17. Satinsky, B. M. et al. Microspatial gene expression patterns in the Amazon River Plume. Proc. Natl. Acad. Sci. U. S. A. 111, 11085-11090 (2014).

18. Franzosa, E. A. et al. Relating the metatranscriptome and metagenome of the human gut. Proc. Natl. Acad. Sci. U. S. A. 111, E2329-38 (2014).

19. Hultman, J. et al. Multi-omics of permafrost, active layer and thermokarst bog soil microbiomes. Nature 521, 208-212 (2015).

20. Maus, I. et al. Unraveling the microbiome of a thermophilic biogas plant by metagenome 
and metatranscriptome analysis complemented by characterization of bacterial and archaeal isolates. Biotechnol. Biofuels 9, 171 (2016).

21. Bremges, A. et al. Deeply sequenced metagenome and metatranscriptome of a biogasproducing microbial community from an agricultural production-scale biogas plant. Gigascience 4, 33 (2015).

22. Shi, Y., Tyson, G. W., Eppley, J. M. \& DeLong, E. F. Integrated metatranscriptomic and metagenomic analyses of stratified microbial assemblages in the open ocean. ISME J. 5, 999-1013 (2011).

23. Dupont, C. L. et al. Genomes and gene expression across light and productivity gradients in eastern subtropical Pacific microbial communities. ISME J. 9, 1076-1092 (2015).

24. Gilbert, J. A. et al. Metagenomes and metatranscriptomes from the L4 long-term coastal monitoring station in the Western English Channel. Stand. Genomic Sci. 3, 183-193 (2010).

25. Sunagawa, S. et al. Ocean plankton. Structure and function of the global ocean microbiome. Science 348, 1261359 (2015).

26. Alberti, A. et al. Viral to metazoan marine plankton nucleotide sequences from the Tara Oceans expedition. Sci Data 4, 170093 (2017).

27. Thrash, J. C. et al. Metabolic Roles of Uncultivated Bacterioplankton Lineages in the Northern Gulf of Mexico ‘Dead Zone’. MBio 8, (2017).

28. Sieradzki, E. T., Ignacio-Espinoza, J. C., Needham, D. M., Fichot, E. B. \& Fuhrman, J. A. Dynamic marine viral infections and major contribution to photosynthetic processes shown by spatiotemporal picoplankton metatranscriptomes. Nat. Commun. 10, 1169 (2019).

29. Baker, B. J., Lesniewski, R. A. \& Dick, G. J. Genome-enabled transcriptomics reveals archaeal populations that drive nitrification in a deep-sea hydrothermal plume. ISME J. 6, 
2269-2279 (2012).

30. Li, M. et al. Genomic and transcriptomic evidence for scavenging of diverse organic compounds by widespread deep-sea archaea. Nat. Commun. 6, 8933 (2015).

31. Lynch, M. D. J. \& Neufeld, J. D. Ecology and exploration of the rare biosphere. Nat. Rev. Microbiol. 13, 217-229 (2015).

32. Faghihi, M. A. \& Wahlestedt, C. Regulatory roles of natural antisense transcripts. Nat. Rev. Mol. Cell Biol. 10, 637-643 (2009).

33. Jousset, A. et al. Where less may be more: how the rare biosphere pulls ecosystems strings. ISME J. 11, 853-862 (2017).

34. Shapiro, B. J. et al. Population genomics of early events in the ecological differentiation of bacteria. Science 336, 48-51 (2012).

35. Arrhenius, S. Über die Reaktionsgeschwindigkeit bei der Inversion von Rohrzucker durch Säuren. Zeitschrift für physikalische Chemie 4, 226-248 (1889).

36. Brown, J. H., Gillooly, J. F., Allen, A. P., Savage, V. M. \& West, G. B. TOWARD A METABOLIC THEORY OF ECOLOGY. Ecology 85, 1771-1789 (2004).

37. Fuhrman, J. A. et al. A latitudinal diversity gradient in planktonic marine bacteria. Proc. Natl. Acad. Sci. U. S. A. 105, 7774-7778 (2008).

38. Allen, A. P., Brown, J. H. \& Gillooly, J. F. Global biodiversity, biochemical kinetics, and the energetic-equivalence rule. Science 297, 1545-1548 (2002).

39. Tilman, D., Mattson, M., Langer - Limnology and Oceanography, S. \& 1981. Competition and nutrient kinetics along a temperature gradient: an experimental test of a mechanistic approach to niche theory. Wiley Online Library (1981).

40. Gillooly, J. F., Allen, A. P., West, G. B. \& Brown, J. H. The rate of DNA evolution: effects 
of body size and temperature on the molecular clock. Proc. Natl. Acad. Sci. U. S. A. 102, 140-145 (2005).

41. Allen, A. P., Gillooly, J. F., Savage, V. M. \& Brown, J. H. Kinetic effects of temperature on rates of genetic divergence and speciation. Proc. Natl. Acad. Sci. U. S. A. 103, 9130-9135 (2006).

42. Yooseph, S. et al. Genomic and functional adaptation in surface ocean planktonic prokaryotes. Nature 468, 60-66 (2010).

43. Lauro, F. M. et al. The genomic basis of trophic strategy in marine bacteria. Proc. Natl. Acad. Sci. U. S. A. 106, 15527-15533 (2009).

44. Giovannoni, S. J., Cameron Thrash, J. \& Temperton, B. Implications of streamlining theory for microbial ecology. ISME J. 8, 1553-1565 (2014).

45. Thompson, A. W. et al. Unicellular cyanobacterium symbiotic with a single-celled eukaryotic alga. Science 337, 1546-1550 (2012).

46. Campbell, B. J., Yu, L., Straza, T. \& Kirchman, D. L. Temporal changes in bacterial rRNA and rRNA genes in Delaware (USA) coastal waters. Aquat. Microb. Ecol. 57, 123-135 (2009).

47. Baker, S. C. et al. The External RNA Controls Consortium: a progress report. Nat. Methods 2, 731-734 (2005).

48. Hutchison, J. S. et al. Hypothermia therapy after traumatic brain injury in children. $N$. Engl. J. Med. 358, 2447-2456 (2008).

49. Bushnell, B. BBMap short read aligner. University of California, Berkeley, California. URL http://sourceforge. net/projects/bbmap (2016).

50. Li, D., Liu, C.-M., Luo, R., Sadakane, K. \& Lam, T.-W. MEGAHIT: an ultra-fast single- 
node solution for large and complex metagenomics assembly via succinct de Bruijn graph.

Bioinformatics 31, 1674-1676 (2015).

51. Jenuth - Bioinformatics Methods and Protocols, J. P. \& 1999. The NCBI: publicly available tools and resources on the web. Springer (1999).

52. Buchfink, B., Xie, C. \& Huson, D. H. Fast and sensitive protein alignment using DIAMOND. Nat. Methods 12, 59-60 (2015).

53. NCBI Resource Coordinators. Database resources of the National Center for Biotechnology Information. Nucleic Acids Res. 44, D7-19 (2016).

54. Kopylova, E., Noé, L. \& Touzet, H. SortMeRNA: fast and accurate filtering of ribosomal RNAs in metatranscriptomic data. Bioinformatics 28, 3211-3217 (2012).

55. Liao, Y., Smyth, G. K. \& Shi, W. featureCounts: an efficient general purpose program for assigning sequence reads to genomic features. Bioinformatics 30, 923-930 (2014).

56. Kim, D., Song, L., Breitwieser, F. P. \& Salzberg, S. L. Centrifuge: rapid and sensitive classification of metagenomic sequences. Genome Res. 26, 1721-1729 (2016).

57. Altschul, S. F., Gish, W., Miller, W., Myers, E. W. \& Lipman, D. J. Basic local alignment search tool. J. Mol. Biol. 215, 403-410 (1990).

58. Van Rossum, G. Python Programming Language. Proc. USENIX Annu. Tech. Conf. (2007).

59. McKinney - Proceedings of the 9th Python in Science, W. \& 2010. Data structures for statistical computing in python. pdfs.semanticscholar.org (2010).

60. Huang, Y., Niu, B., Gao, Y., Fu, L. \& Li, W. CD-HIT Suite: a web server for clustering and comparing biological sequences. Bioinformatics 26, 680-682 (2010).

61. Koslicki, D. \& Falush, D. MetaPalette: a k-mer Painting Approach for Metagenomic Taxonomic Profiling and Quantification of Novel Strain Variation. mSystems 1, (2016). 
bioRxiv preprint doi: https://doi.org/10.1101/636373; this version posted May 16, 2019. The copyright holder for this preprint (which was not certified by peer review) is the author/funder, who has granted bioRxiv a license to display the preprint in perpetuity. It is made available under aCC-BY-NC-ND 4.0 International license.

62. Team, R. C. R language definition. Vienna, Austria: R foundation for statistical computing (2000).

63. Dixon, P. VEGAN, a package of R functions for community ecology. J. Veg. Sci. 14, 927930 (2003). 


\section{Supplementary information}
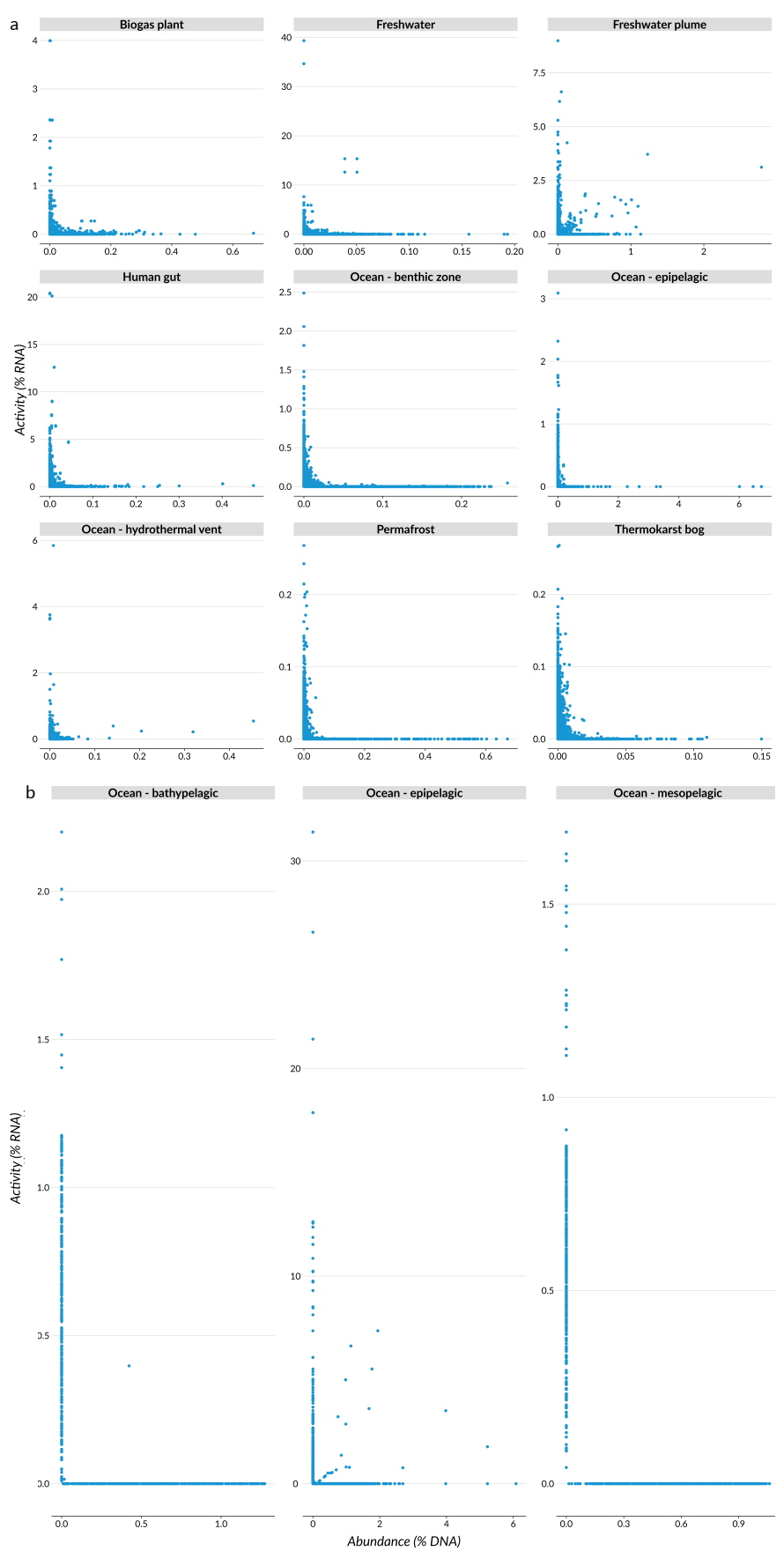

Figure S1. Relationships between relative abundance (\% DNA) and activity (\% RNA).

Relationships between relative abundance and activity regardless of taxonomy that were sequenced with the a) Illumina and b) Roche 454 platforms. 


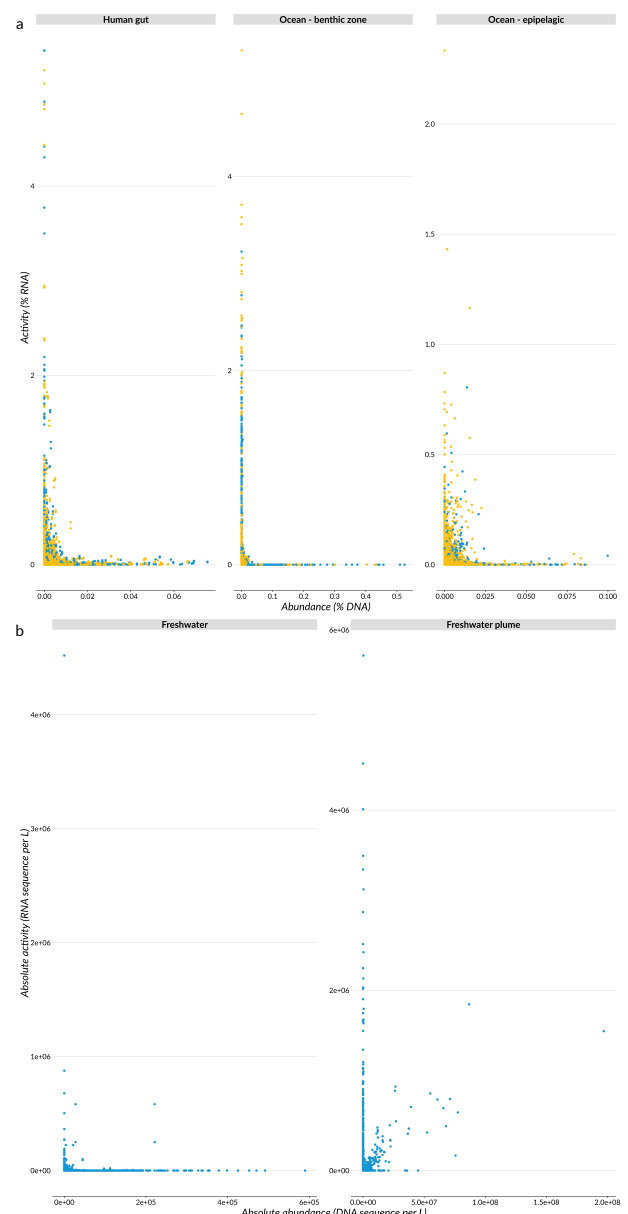

Figure S2. Relationships between abundance and activity for samples sequenced with methods that retain RNA strand direction and absolute quantifications. a) Stranded (directional) relationships between relative abundance and relative activity. Strandedness is only retained at the ORF level therefore mappings were counted at the ORF level. The sense (yellow) reflects transcripts that directly correspond to mRNA that will be translated into peptides. Antisense (blue) transcripts directly correspond to transcripts that are the reverse complement of mRNA and can act as transcriptional and post-translational regulators ${ }^{1}$. b) Relationships between absolute abundance (DNA sequence $\mathrm{L}^{-1}$ ) and absolute activity (RNA sequence $\mathrm{L}^{-1}$ ) from the Amazon River ${ }^{2}$ and the Amazon River plume ${ }^{3,4}$. Absolute quantities were generated by multiplying relative frequencies by measured quantities of DNA and RNA from Satinsky et al., $2014 \mathrm{a}^{19}$, Satinsky et al., 2014b $\mathrm{b}^{20}$, Satinsky et al., 2015 ${ }^{18}$. Briefly, DNA and RNA samples were amended with known quantities at the time of nucleic acid extraction. The percent recovery of the DNA and RNA additions provide a direct conversion of relative frequencies. 

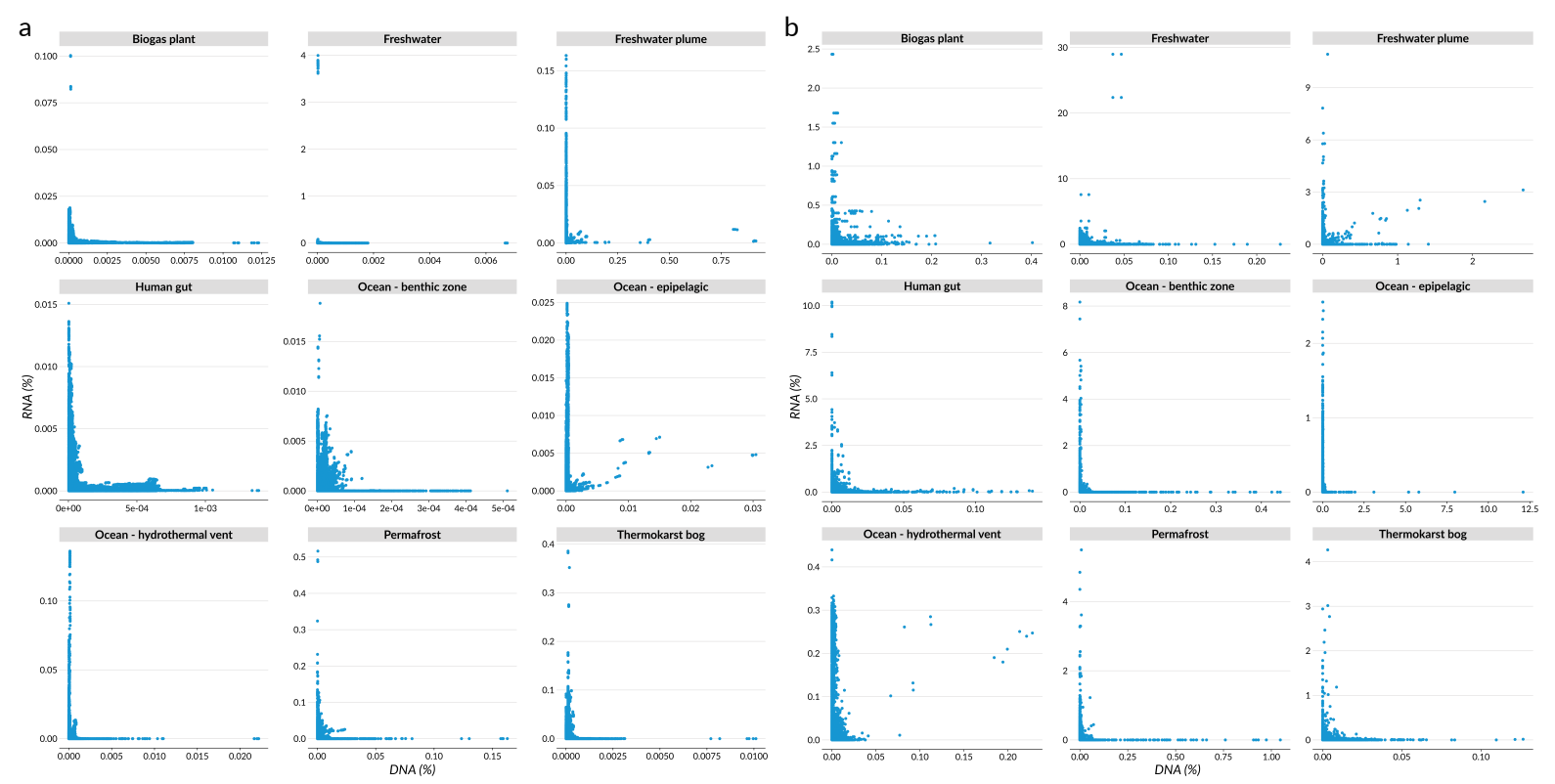

c
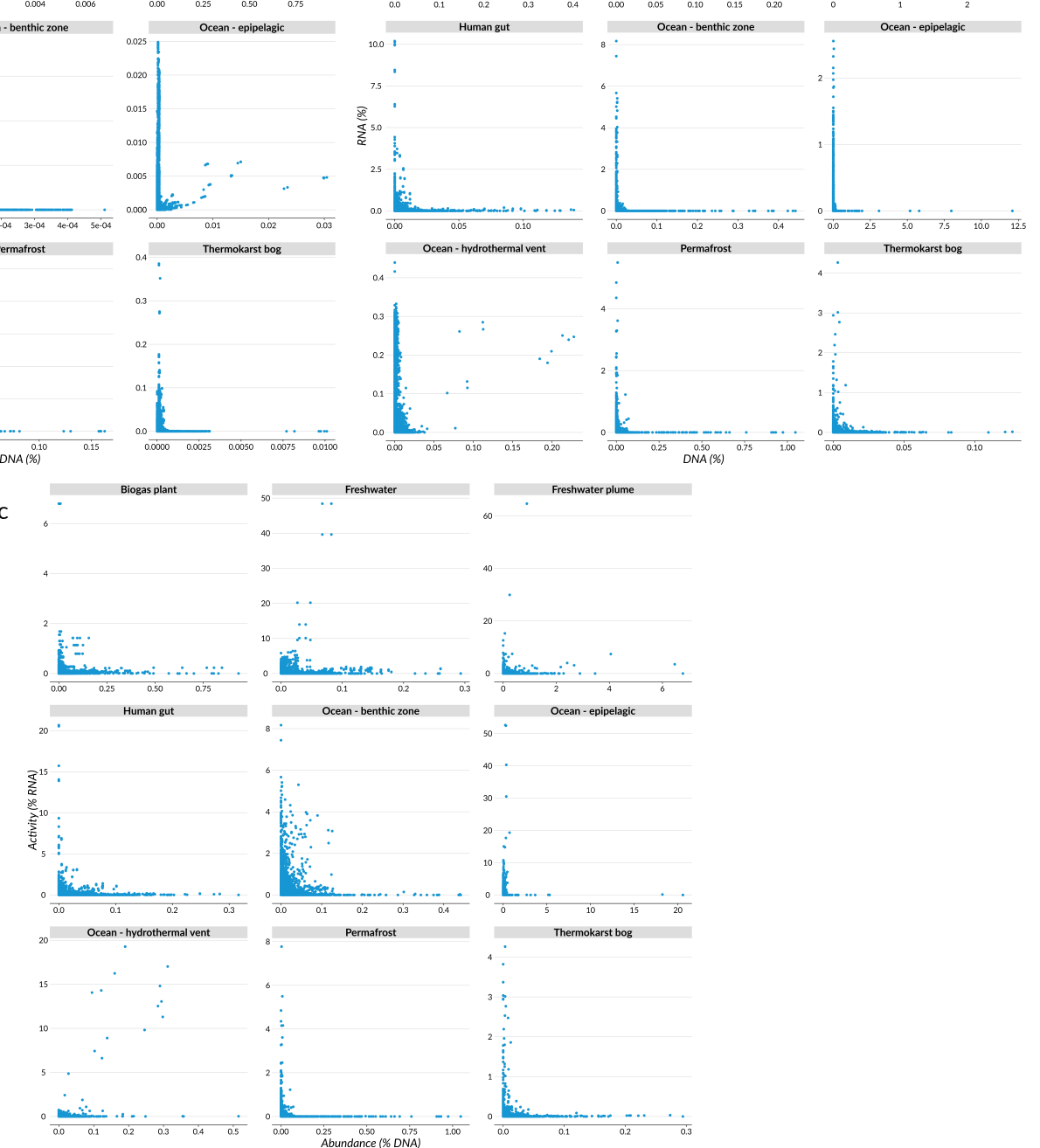

Figure S3. Relationships between relative abundance (\% DNA) and relative activity (\% RNA) of kmers, ORFs, and approximate functional clusters. a) Abundance and activity of microbial communities using kmer counts. Kmers with low complexity were removed and only those with counts $>2$ per sample that were represented in both DNA and RNA fractions were retained. b) Relationships between abundance and activity of ORFs. c) Abundance and activity of functional protein clusters were generated by semi-global clustering of ORFs at $60 \%$ identity. Relative frequencies for each cluster were generated by summing the relative frequencies of ORFs that formed each cluster. 


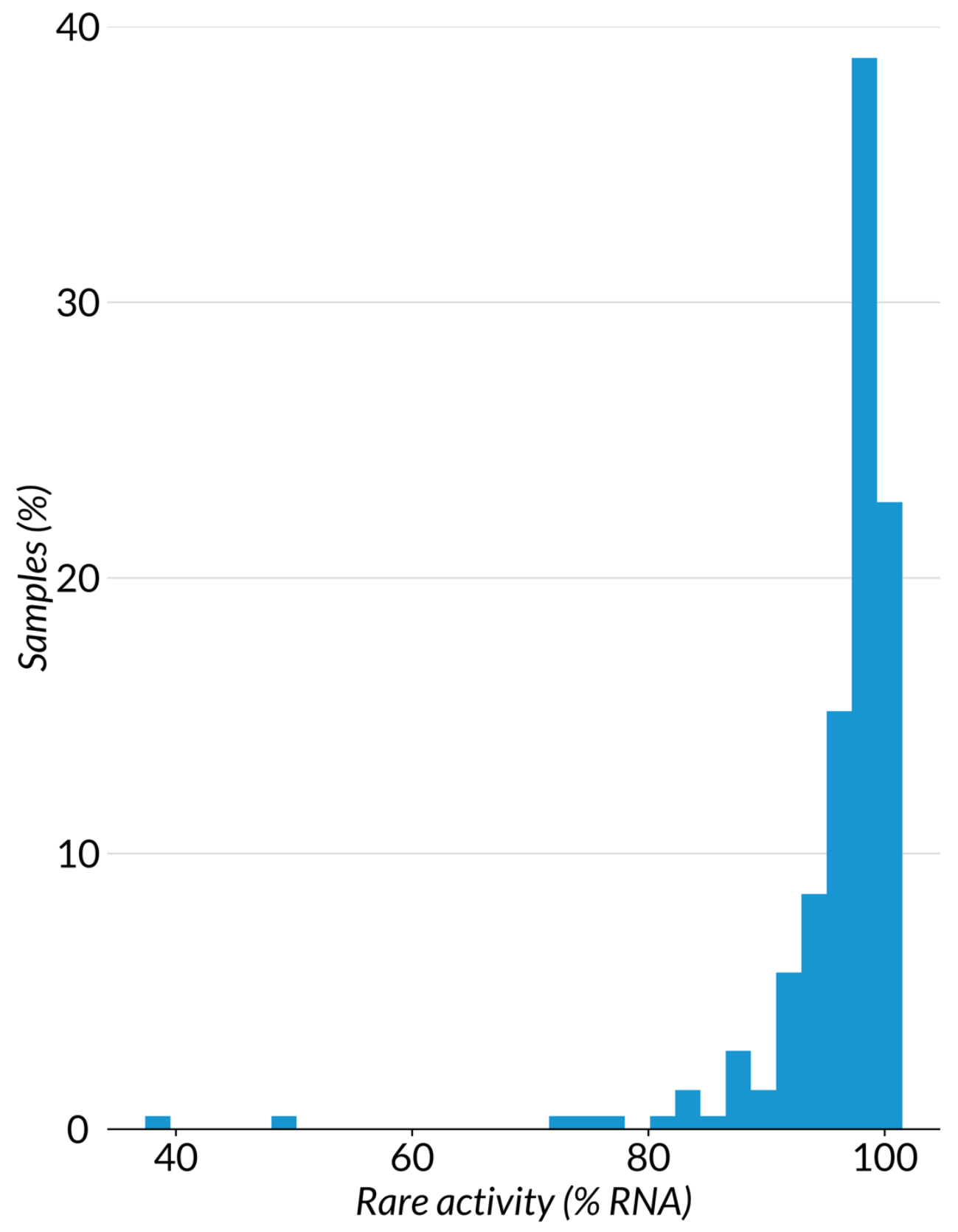

Figure S4. Distribution of rare activity across all Illumina sequenced samples. Rare activity is expressed as the relative RNA frequencies of assemblies in the rare fraction. 
a
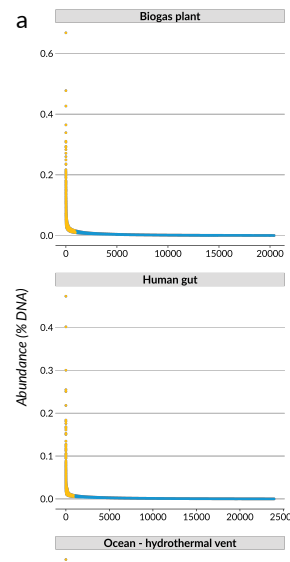

0.4

$0.3-$

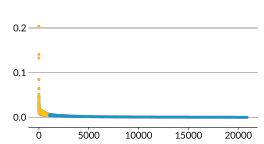

$\mathrm{b}_{4}$
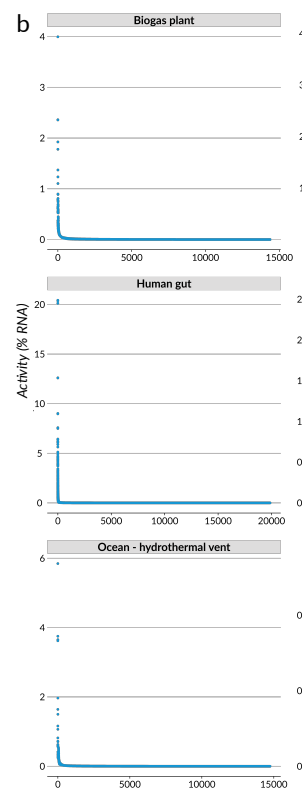
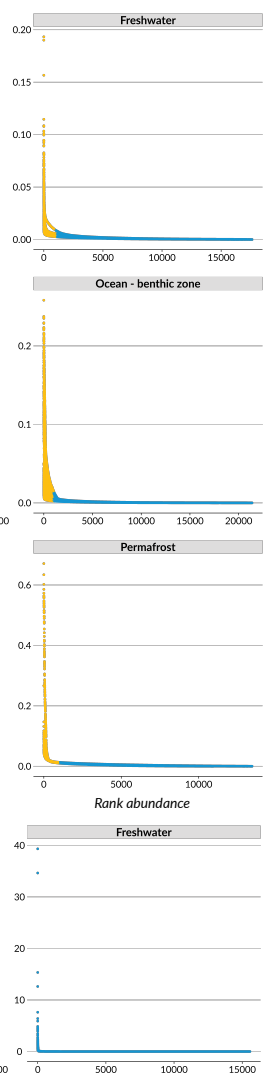

ccean - benthic zone
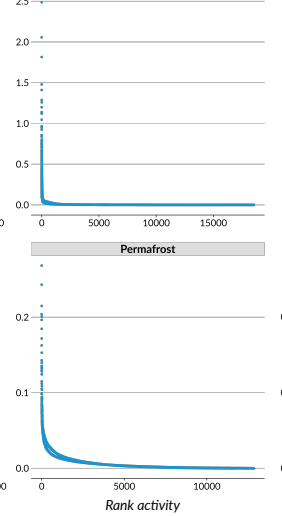
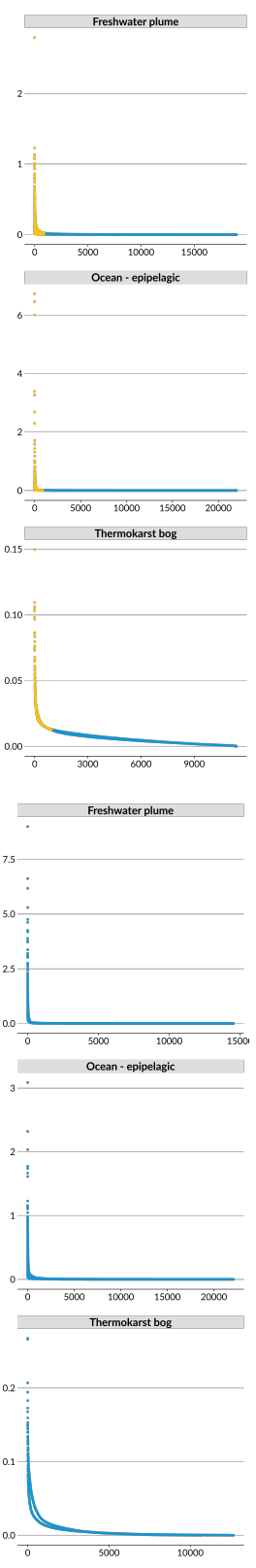

Figure S5. Relative abundance (\% DNA) as a function of DNA rank abundance of metagenomic and metatranscriptomic sequence assemblies for Illumina sequenced samples. a) Rank abundance curves by environment. Rare assemblies are colored blue and abundant assemblies are yellow. Rare is considered $>1000$ th rank and are in the tail of the rank abundance curves or a mean of $0.005 \%$. b) Rank activity curves by environment. Rank activity is plotted as activity (\% RNA) as a function of rank activity. 
a

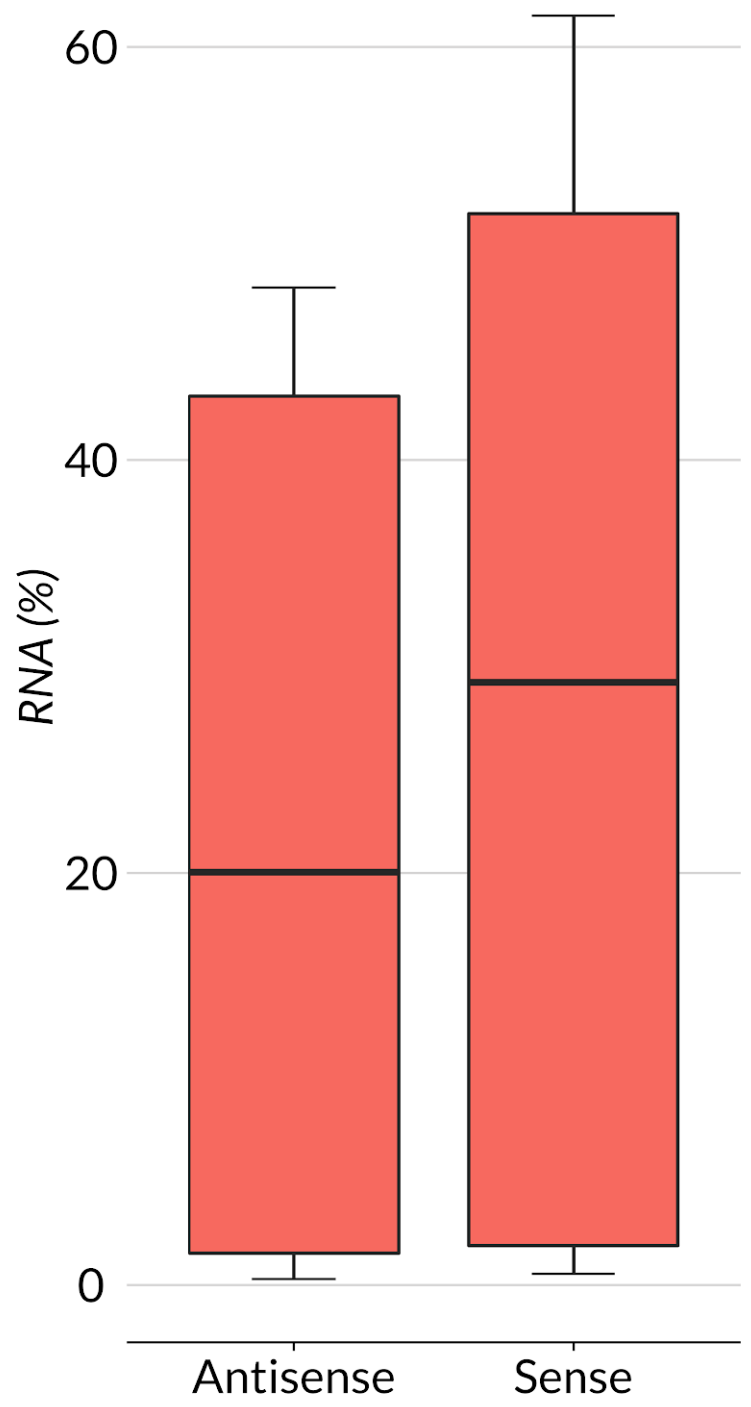

b

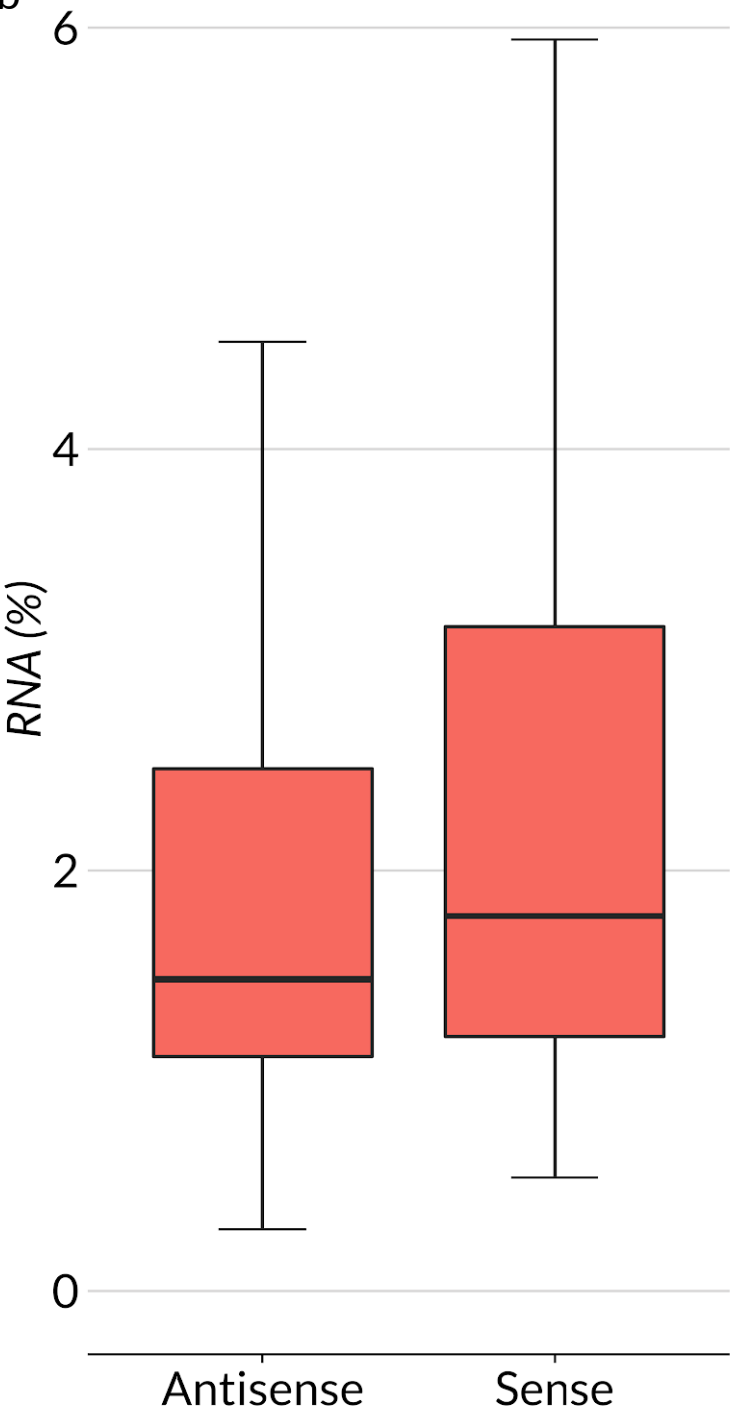

Figure S6. Comparison between antisense and sense expression in whole and rare

community fractions. a) Box and whisker plot of antisense and sense rare microbial activity $(P$ $\left.=3.6 \times 10^{-6}\right)$. b) Box and whisker plot of antisense and sense whole community activity $(P=$ 0.1 ). 

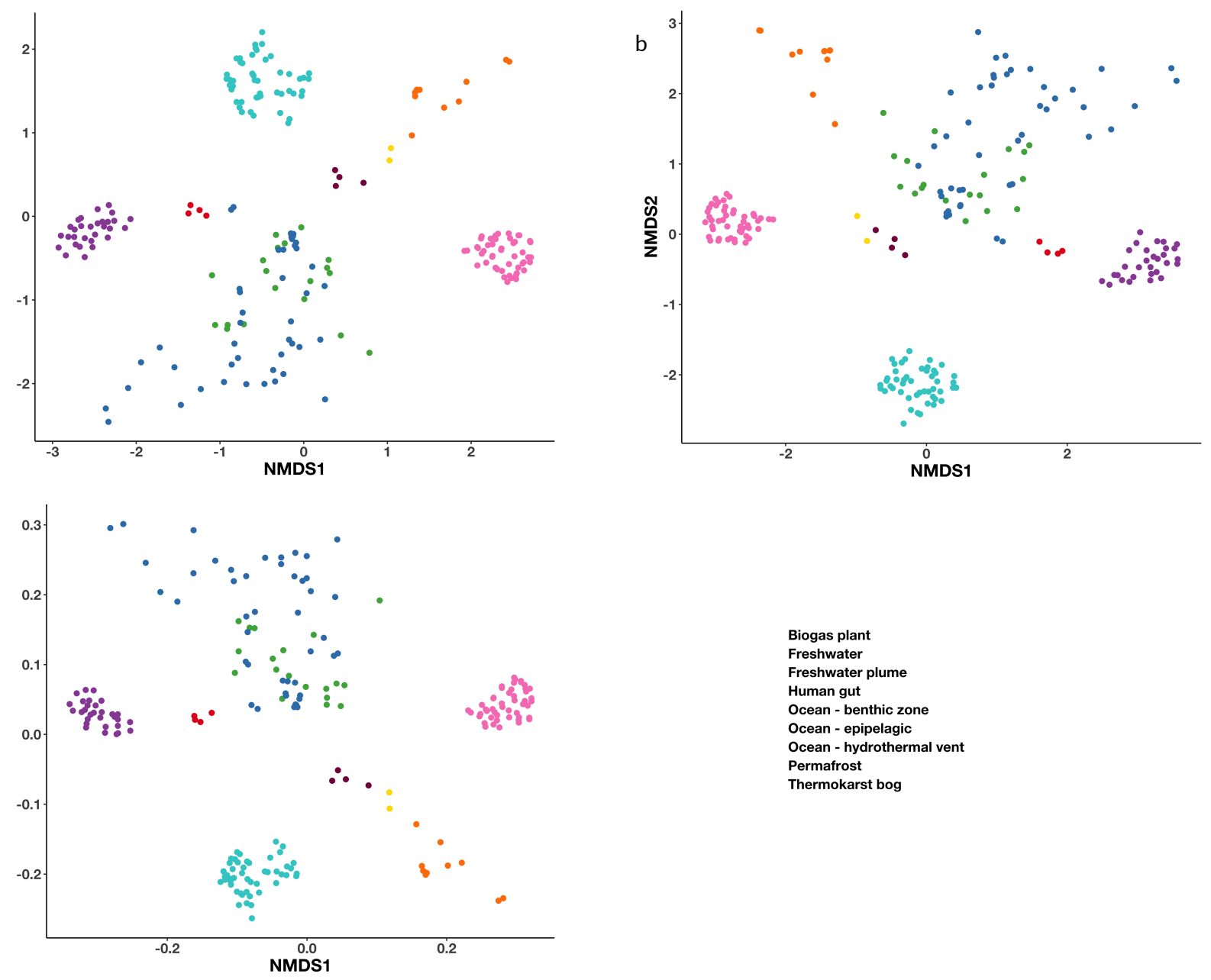

Figure S7. Non-metric multidimensional scaling (NMDS) based ordination of samples by

environment. Sample distances are based on Bray-Curtis dissimilarities of a) abundance (\% DNA) b) activity (\% RNA) c) specific activity (RNA:DNA ratios). 


\section{0}

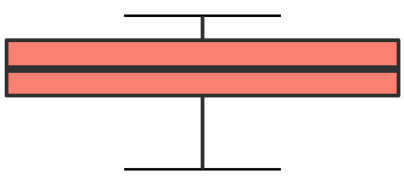

0.9

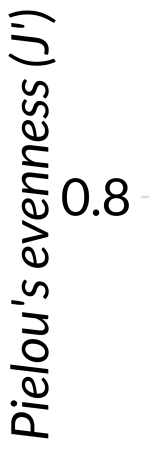

0.7

0.6

\section{DNA}

\section{RNA}

Figure S8. Box and whisker plots of abundance (DNA) and expression (RNA) evenness across all Illumina sampled sequences. Evenness is calculated as Pielou's evenness $\left(J^{\prime}\right)$ of sequence assemblies and their relative frequencies in metagenomes and metatranscriptomes (Mann-Whitney $U$ test; $P<2.2 \times 10^{-16}$ ). 


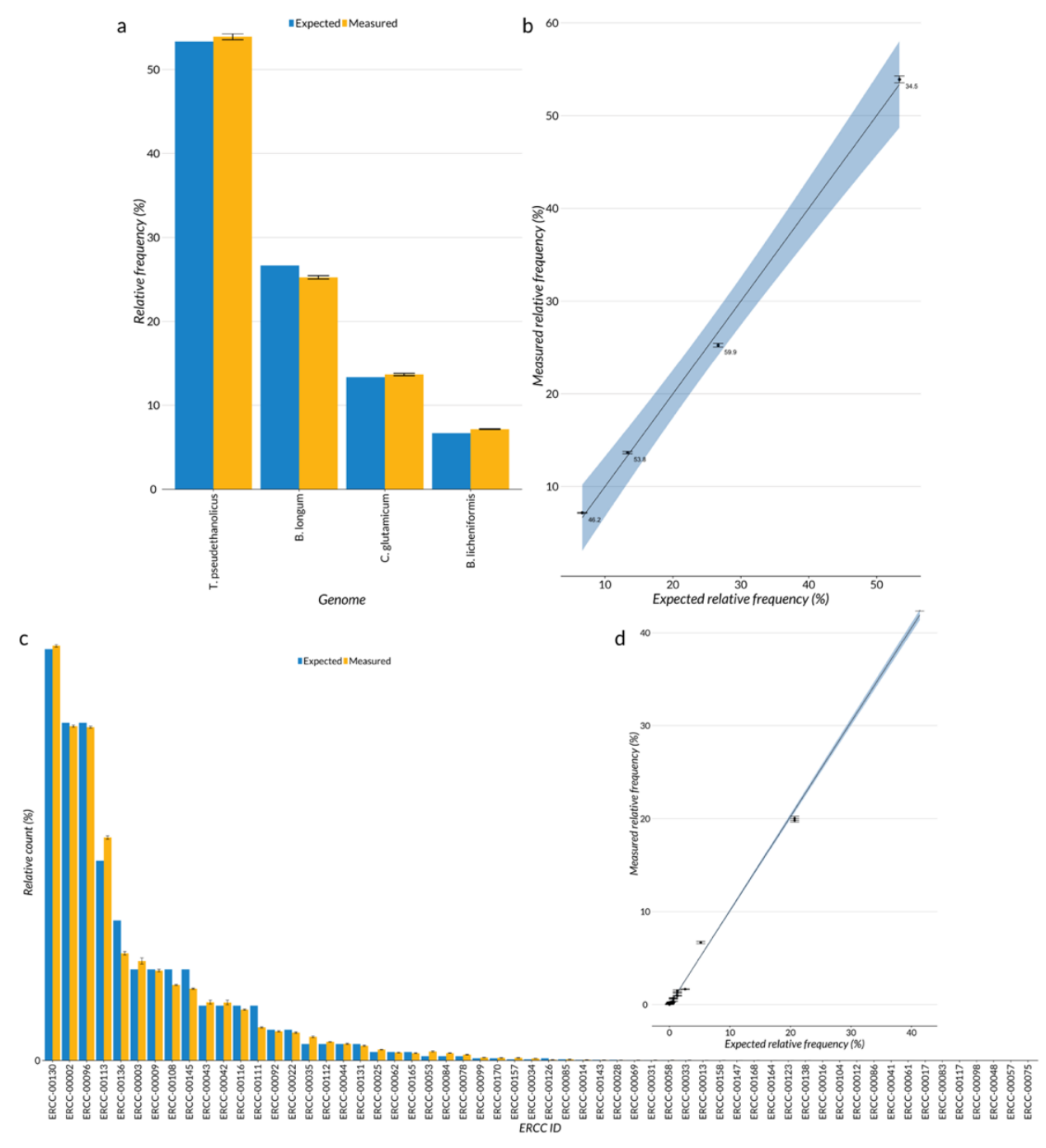

Figure S9. Evaluation of control sequences. a) Mean measured and relative frequencies from SPOT benthic zone metagenomes for each amended genome control with SEM $(n=32)$. b) Linear regression of measured metagenomic relative frequencies against expected relative frequencies of SPOT benthic zone amended genome controls plotted with $95 \%$ confidence interval and $\operatorname{SEM}\left(n=32 ; R^{2}=0.9933\right)$. c) Mean measured and relative frequencies from SPOT benthic zone metatranscriptomes for each $\operatorname{ERCC}$ control with SEM $(n=30)$. d) Linear regression of measured metatranscriptomic relative frequencies against expected relative frequencies of SPOT benthic zone ERCC controls plotted with 95\% confidence interval and SEM $\left(n=30 ; R^{2}=\right.$ $0.9932)$ 
Table S1. Data sources used in this study (see excel table). Most data sources are provided by NCBI SRA or ENA accession numbers. Sequence libraries are characterized by nucleic acid source (DNA or RNA), sequencing technology, preparation methods, source environment, attached/free lifestyle, and sample temperature. Pair bin denotes matching DNA and RNA samples. Pairing information was retrieved from the data source databases and source publications. Public data were retrieved from: Alberti et al., 201729, Baker et al., 2012 32 ,

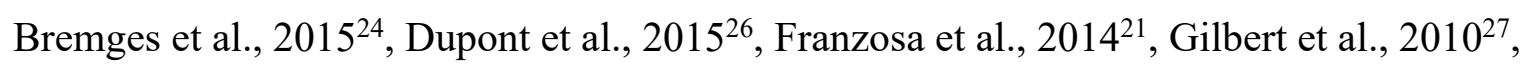
Hultman et al., 2015 22 , Li et al., 2015 33, Maus et al., 2016 ${ }^{23}$, Satinsky et al., 2014a ${ }^{19}$, Satinsky et al., $2014 b^{20}$, Satinsky et al., 2015 ${ }^{18}$, Shi et al., $2011^{25}$, Sieradzki et al., 2017 $7^{30}$, Sunagawa et al., $2015^{28}$, and Thrash et al., $2017^{31}$.

Table S2. RNA contribution of rare and abundant microbes (see excel table). \% RNA of rare and abundant microbes was totaled for each sample sequenced on Illumina platforms. Total $\%$ RNA across samples was calculated by summing \% RNA for across all samples and renormalizing to the summed \% RNA for each abundance fraction.

Table S3. Annotations of the most expressed ORFs (see excel table). RefSeq and KEGG annotations of the most expressed ORFs from each sample pairing that had a RefSeq match. 


\begin{tabular}{|c|c|c|c|c|}
\hline $\begin{array}{l}\text { NCBI } \\
\text { Accession }\end{array}$ & $\begin{array}{l}\text { Genome } \\
\text { name }\end{array}$ & $\begin{array}{l}\% \\
\text { G+ } \\
\text { C }\end{array}$ & Fol & NCBI Taxonomy \\
\hline NC_006322 & $\begin{array}{l}\text { Bacillus } \\
\text { lichenifor } \\
\text { mis DSM } \\
13= \\
\text { ATCC } \\
14580\end{array}$ & 46.2 & 1 & $\begin{array}{l}\text { Bacteria; Firmicutes; Bacilli; Bacillales; Bacillaceae; } \\
\text { Bacillus }\end{array}$ \\
\hline NC_011593 & $\begin{array}{l}\text { Bifidobact } \\
\text { erium } \\
\text { longum } \\
\text { subsp. } \\
\text { infantis } \\
\text { ATCC } \\
15697\end{array}$ & 59.9 & 4 & $\begin{array}{l}\text { Bacteria; Actinobacteria; Bifidobacteriales; } \\
\text { Bifidobacteriaceae; Bifidobacterium }\end{array}$ \\
\hline NC_ 003450 & $\begin{array}{l}\text { Corynebact } \\
\text { erium } \\
\text { glutamicu } \\
\text { m ATCC } \\
13032\end{array}$ & 53.8 & 2 & $\begin{array}{l}\text { Bacteria; Actinobacteria; Corynebacteriales; } \\
\text { Corynebacteriaceae; Corynebacterium }\end{array}$ \\
\hline NC_010321 & $\begin{array}{l}\text { Thermoana } \\
\text { erobacter } \\
\text { pseudethan } \\
\text { olicus } \\
\text { ATCC } \\
33223\end{array}$ & 34.5 & 8 & $\begin{array}{l}\text { Bacteria; Firmicutes; Clostridia; } \\
\text { Thermoanaerobacterales; Thermoanaerobacteraceae; } \\
\text { Thermoanaerobacter }\end{array}$ \\
\hline
\end{tabular}

Table S4. Genome controls used to amend SPOT metagenomes. Each genome was selected to encompass a range of $\% \mathrm{G}+\mathrm{C}$. 